\title{
Tuning of nanostructures of gold nanoparticles on indium tin oxide surfaces using a seed-mediated growth method
}

$\operatorname{AUTHOR}(S):$

Kajita, Tomonori; Oyama, Munetaka

\section{CITATION:}

Kajita, Tomonori ... [et al]. Tuning of nanostructures of gold nanoparticles on indium tin oxide surfaces using a seed-mediated growth method. Journal of Electroanalytical Chemistry 2011, 656(1-2): 264-268

ISSUE DATE:

2011-06

URL:

http://hdl.handle.net/2433/159445

\section{RIGHT:}

(c) 2010 Elsevier B.V.; この論文は出版社版でありません。引用の際には 出版社版をご確認ご利用ください。; This is not the published version. Please cite only the published version. 
Revised Manuscript for Journal of Electroanalytical Chemistry for the special issue in honour of S. Dong

\title{
Tuning of nanostructures of gold nanoparticles on indium tin oxide surfaces using a seed-mediated growth method
}

\author{
Tomonori Kajita and Munetaka Oyama ${ }^{*}$ \\ Department of Material Chemistry, Graduate School of Engineering, \\ Kyoto University, Nishikyo-ku, Kyoto 615-8520, Japan. \\ Tel: $\quad+81-75-383-3074, \quad$ Fax: $+81-75-383-3074$ \\ E-mail: m.oyama@kx8.ecs.kyoto-u.ac.jp
}

* To whom correspondence should be addressed. 


\section{Abstract}

Tuning of nanostructutres of gold nanoparticles (AuNPs) on indium tin oxide (ITO) surfaces could be performed using a seed-mediated growth method. While this method composed of two steps, i.e., a seeding process and a growth process, it was found that the smaller AuNPs with a higher density grew on ITO by adding hexamethylenetetramine (HMT) into a growth solution, which normally contained cetyltrimethylammonium bromide (CTAB) as a capping reagent. The optimal conditions for preparing the denser AuNPs with higher size uniformity were found that the ratio of CTAB:HMT was 1:1. For the electrochemical oxidation of uric acid, the AuNP-attached ITO electrode prepared at the 1:1 ratio of CTAB:HMT had superior electrocatalytic properties to the AuNP-attached ITO electrode prepared with the previous growth solution, i.e., CTAB only. The effects of the nanostructures of AuNPs on the electrocatalytic properties were examined after further tuning, i.e., by repeating the growth treatments. As the result, it was found that an increase in the amount of gold not necessarily improve the electrocatalytic properties, but the smaller and denser attachment prepared with HMT was effective for the case of the oxidation of uric acid. The electrochemical oxidations of ascorbic acid and epinephrine showed the same tendencies.

Key words: Gold nanoparticles, electrocatalytic properties, indium tin oxide, uric acid, nanostructured surfaces. 


\section{Introduction}

Metal nanoparticles (NPs) modified electrodes have been attracting active attention in recent years [1-3]. Nanostructuring on the electrode surfaces with metal NPs can be achieved with electrochemical and chemical methods, and the thus-prepared surfaces have been successfully applied to electroanalysis [1-3].

While the electrochemical results tend to be reported using individually-prepared metal NPs modified electrodes, some comparisons would be necessary for understanding electrocatalytic properties depending on the nanostructures of metal NPs on the surfaces. Thus, a concept of tuning of nanostructures of metal NPs on conducting substrates would be important for fabricating metal NPs modified electrodes having better performances. In the electrochemical preparations, the tuning of the metal nanostructures would be relatively easy with the control of the conditions as reported for the cases of gold NPs (AuNPs) on glassy carbon $[4,5]$ and indium tin oxide (ITO) surfaces [6,7]. However, in the chemical preparations, some post-treatments are necessary if we assume the conventional attachment of chemically synthesized metal NPs with linker molecules.

For such chemical tunings, Brown and Natan reported a chemical reduction with hydroxylamine valid for enlargement of surface-confined colloidal AuNPs [8]. Dong and coworker reported the controlled nucleation and growth of surface confined AuNPs [9]. By combining the growth with the colloid chemical approach to nanoelectrode ensembles [10], a fine tuning of the nanoelectrode ensembles were reported. [11].

Following these pioneering works, our group is interested in the nanostructural growth from nanoseed particles [12-22]. Interestingly, smaller NPs tend to attach on the ITO surfaces via physi-sorption, and therefore, gradual structural growth is possible 
in the following treatment [12]. The thus-prepared AuNP-attached ITO electrodes could be applied to electroanalysis [13-16], and some controls of the grown nanostructure of Au were possible by revising the seeding procedures [17-19] and the growth process [20]. In addition, this seed-mediated growth approach for surface modification was found to be valid for the cases of AgNPs [21] and PdNPs [22] on ITO substrates.

In the present paper, we would like to show that tuning of the grown nanostructure of AuNPs is possible using an additive reagent in the growth solution. Actually, hexamethylenetetramine (HMT) was added into the growth solution, which originally contained CTAB only as a capping reagent in our previous works. HMT is water-soluble amine, and used as an additive to form $\mathrm{ZnO}$ nanorods on substrates [23]. In addition, the mixed solutions of CTAB and HMT were shown to be an interesting media to form Pd nanobricks [24] and Au nanotripods [25].

Since the same mixed solutions allowed us the tuning of the nanostructures of AuNPs, we compare the electrocatalytic properties by recording cyclic voltammograms of uric acid. In the previous papers [13,14, 26, 27], it has been reported that AuNP-attached ITO electrodes are effective for electrocatalytic oxidation of uric acid. In addition, for further tuning of surface structure, some repeated growth treatments are reported to compare the electrocatalytic properties.

\section{Experimental}

\subsection{Apparatus and materials}

Scanning electron microscopic (SEM) images were obtained with a field emission SEM instrument (JSM-7400F, JEOL, Japan). Electrochemical experiments were 
carried out with an EG\&G M263A potentiostat/galvanostat (Princeton Applied Research, USA) controlled by M270 programs. Glass plates on which ITO was sputtered (10 mm x $26 \mathrm{~mm} \times 1.0 \mathrm{~mm})$ were the products of CBC Optics Co. Ltd., and used after washing in acetone, ethanol and pure water with sonication for 15 minutes, and drying with nitrogen gas. In the electrochemical measurements, a bare or modified ITO electrode was fabricated to exposure the 2-mm diameter area was a working electrode. A platinum wire and an $\mathrm{Ag} / \mathrm{AgCl}(3 \mathrm{M} \mathrm{NaCl})$ electrode were employed as the counter and reference electrodes, respectively.

Cetyltrimethyammonium bromide (CTAB), hexamethylenetetramine (HMT) and $\mathrm{HAuCl}_{4} \cdot 3 \mathrm{H}_{2} \mathrm{O}$ were purchased from Aldrich Co. Ltd. Other reagents were obtained from Wako Pure Chemicals Ltd. All solutions were prepared with ultra-pure water obtained from a water purification system (Millipore WR600A, Yamato Co., Japan).

\subsection{Seed-mediated growth method for modifying AuNPs on ITO surfaces}

While smaller Au nanoseed particles of ca. $4 \mathrm{~nm}$ tend to attach on ITO surfaces by just immersing the substrates into the solution [12], an in situ reduction [15] was carried out in the present work to ensure the first attachment. As the actual procedures, in the first seeding process, an ITO substrate was immersed in a solution composed of $0.5 \mathrm{~mL}$ of $0.01 \mathrm{M} \mathrm{HAuCl}_{4}, 0.5 \mathrm{~mL}$ of $0.01 \mathrm{M}$ trisodium citrate and $20 \mathrm{~mL} \mathrm{H}_{2} \mathrm{O}$. After leaving for $15 \mathrm{~min}, 0.5 \mathrm{~mL}$ of ice-cold $0.1 \mathrm{M} \mathrm{NaBH}_{4}$ aqueous solution was added into the solution, and the solution was left undisturbed for $2 \mathrm{~h}$. Next, the substrate was immersed in growth solution after thoroughly rinsed with distilled water and dried with nitrogen. The general growth solution was prepared by mixing $20 \mathrm{~mL}$ of $0.10 \mathrm{M}$ CTAB, $0.5 \mathrm{~mL}$ of $0.01 \mathrm{M} \mathrm{HAuCl}_{4}, 0.1 \mathrm{~mL}$ of $0.1 \mathrm{M}$ ascorbic acid and $0.1 \mathrm{~mL}$ of $0.1 \mathrm{M}$ 
$\mathrm{NaOH}$ solution. In the present work, we observe the effects of HMT in the growth solution. For this purpose, the concentrations of [CTAB] and [HMT] were changed by keeping the sum of $[\mathrm{CTAB}]$ and $[\mathrm{HMT}]$ as $0.10 \mathrm{M}$. The immersing time into the growth solution was typically 24 hours. However, the grown structures were not significantly altered after 3 hours in the case of the 1:1 mixied solution of CTAB:HMT, we adopted 3 hours' growth in some repeated treatments.

\section{Results and Discussion}

\subsection{Effects of HMT on the nanostructures of AuNPs on ITO surfaces}

Figure 1 shows FE-SEM images of AuNP-modified ITO surfaces prepared using the growth solution containing both CTAB and HMT. Here, keeping the total concentrations of $[\mathrm{CTAB}]+[\mathrm{HMT}]$ in the growth solution as $0.10 \mathrm{M}$, the changes in the FE-SEM images were observed. As the result, remarkable transformation of the surface images could be observed depending on the mixing ratio as summarized in Fig.1.

As a noticeable outcome, when the ratio of CTAB:HMT was 1:1, smaller AuNPs whose size was ca. $30 \mathrm{~nm}$ were observed to have grown up densely on the ITO surface (Fig. 1D). The density was ca. $1.5 \times 10^{3}$ particles $/ \mu \mathrm{m}^{2}$. When the amount of CTAB is larger than that of HMT, typically at 3:1 (Fig. 1A), some larger AuNPs of ca. $200 \mathrm{~nm}$ were observed to grow, which is due to a kind of Ostwald ripening to grow lager AuNPs with the dissolution of smaller AuNPs. Thus, a suitable amount of HMT is expected to have interacted with gold ions in the growth solution, and altered the reductive growth process of AuNPs. As the result of the strong interaction between HMT and gold ions, it is considered that smaller AuNPs of ca. $30 \mathrm{~nm}$ grew densely on 
the ITO surface as shown in Fig. 1D. While $\mathrm{I}^{-}$impurity has been recently reported to affect the formation of gold nanorods in solution [28, 29], the equimolar amount of HMT is reasonably expected to alter the capping actions of CTAB during the crystal growth of AuNPs.

However, when the concentration of HMT is larger than that of CTAB, the number of attached AuNPs was quite smaller as shown in Fig. 1E and F. This is probably due to the increased interaction of HMT with gold ions, which could not allow the growth from the nanoseeds.

As shown in Fig. 1, tuning of the nanostructures of AuNPs on ITO surfaces was possible by simply changing the molar ratio of CTAB:HMT in the growth solutions. The same effect of HMT was observed when it was added in the growth solutions in that a constant amount $(0.10 \mathrm{M})$ of CTAB was presented. However, since the changes in the surface images were more noticeable under the conditions of Fig.1, we showed the results, and chose the typical conditions to prepare densely attached smaller AuNPs with higher size uniformity as the 1:1 ratio of CTAB:HMT, i.e., 0.050 M each.

\subsection{Electrocatalytic properties for the oxidation of uric acid.}

Because the densely attached smaller AuNPs with size uniformity could be prepared at the 1:1 ratio of CTAB:HMT, the electrochemical responses were evaluated by observing cyclic voltammograms for the oxidation of uric acid in $0.10 \mathrm{M}$ phosphate buffer solution ( $\mathrm{pH} 7.0)$. Fig.2 shows the cyclic voltammograms measured with (a) a bare ITO electrode, (b) an AuNP-attached ITO electrode prepared with CTAB only, and (c) an AuNP-attached ITO electrode prepared with the 1:1 mixed solution of CTAB and HMT. 
While the oxidation of uric acid with the bare ITO electrode showed smaller oxidation currents at higher potential region as shown in Fig. 2 (a), the onset of the oxidation were much improved to occur at less positive potentials with the AuNP-attached electrodes (Fig. 2 (b) and (c)). In addition, the oxidation currents values increased significantly. Thus, superior electrocatalytic property of the AuNP-attached ITO electrode prepared with the 1:1 ratio of CTAB:HMT. It is inferred that the nanostructured surface of Fig. 1D should be suitable for the electrocatlytic oxidation of uric acid.

\subsection{Further tuning of nanostructures of AuNPs on ITO surfaces.}

Because the AuNP-attached ITO electrode prepared with the 1:1 ratio of CTAB:HMT has found to exhibit better electrocatalytic property, we tried some further tunings of the nanostructures on ITO surfaces. As one trial, the growth in the 1:1 mixed solution of CTAB:HMT was repeated after the first treatment to see what surface was formed. As the result, the surface whose FE-SEM image is shown in Fig. 3A was prepared. This indicates the repeated treatment did not change the surface drastically but slightly increased the numbers of smaller AuNPs attached on the ITO surface. The treatment with the 1:1 solution of CTAB:HMT is considered to do not allow the growth of AuNPs already existed, but tend to promote further growth of smaller AuNPs.

On the other hand, the growth treatment with CTAB only was performed after the first treatment with the 1:1 solution of CTAB:HMT. As the consequence, the surface whose FE-SEM image is shown in Fig. 3B was prepared. As apparent from the figure, the second treatment with the growth solution contained CTAB only brought about a significant increase in the size of AuNPs, typically more than $60 \mathrm{~nm}$, and caused some 
specific connecting structures of grown AuNPs. This result would indicate that this repeated treatment with the growth solutions contained CTAB only should be effective to prepare AuNP-modified surfaces with increased loaded amounts of Au.

\subsection{Electrocatalytic properties of some nanosrtuctured surfaces.}

The electrocatalytic properties of the thus-prepared two surfaces were examined for the case of the oxidation of the uric acid. The observed cyclic voltammograms are shown in Fig. 4. As the result, the electrocatalytic property of the surface of Fig. 3A (Fig. 4a)) was apparently higher than that of the surface of Fig. 3B (Fig. 4b). Moreover, a similarity of the responses was observed between the surfaces prepared CTAB/HMT once (Fig. 2c) and CTAB/HMT twice (Fig. 4a), as well as between the surfaces prepared CTAB once (Fig. 2b) and CTAB/HMT first followed by the treatment with CTAB (Fig. 4b). (Hereafter, CTAB/HMT means the 1:1 mixed solution of

\section{CTAB:HMT.)}

Therefore, the results imply that some electrocatalytic properties might be affected the final treatment in the present cases. The increase of the loaded amount of $\mathrm{Au}$ as shown in Fig. 3B was not necessarily to enhance the electrocatalytic properties of uric acid, but rather the smaller and denser attachment of AuNPs with higher size uniformity favored electrocatalytic oxidation of uric acid.

Following the result that the twice repeated treatment with CTAB/HMT brought about the surface having the best electrocatalytic property, we carried out the experiment for the increased repeated times. Fig. 5 shows the FE-SEM images, and Fig. 6 the cyclic voltammograms of uric acid. The nanostructures of the prepared surfaces were not significantly different from that prepared CTAB/HMT twice, though 
some minor changes in the morphology of gathered AuNPs on ITO surfaces were observed as shown in Fig. 5. As well, while some increases in the electrocatalytic properties were observed compared with the surface prepared CTAB/HMT twice as shown in Fig. 6, the degree of the improvement was not significant in comparison with those from the CTAB-treated surfaces.

The present result may imply that the surfaces prepared with the repeated $\mathrm{CTAB} / \mathrm{HMT}$ treatment exist in a certain higher electrocatalytic level, and therefore, further increase might be not easy. Because the changes in the surface nanostructures were not significant in the repeated treatment, other trials would be necessary to examine the possibility of dramatic increases in the electrocatalytic properties.

Although the responses of the electrooxidation of uric acid were focused on in the present paper, we also carried out some electrochemical measurements of ascorbic acid and epinephrine. As the results, a similar tendency was observed for the order of the electrocatalytic properties. Therefore, the present approach using the growth solution of CTAB/HMT is inferred to be effective for preparing AuNP-modified electrode having higher electrocatalytic properties. The lesser amounts of AuNPs loaded on the surface should be another advantage of the proposed electrodes.

\section{Conclusions}

In the present work, we prepared the ITO surfaces on which densely attached smaller AuNPs with higher size uniformity were densely attached by using HMT as a coexisting reagent in the growth solution. Because HMT is reasonably expected to alter the interactions with cationic species in the growth solution as an amine, the previous growth process with $\mathrm{CTAB}$ as a sole capping reagent was significantly revised 
to allow the new nanostructural growth of AuNPs .

Thus-enabled tuning of nanostructures allowed us to explore the changes in the electrocatalytic properties, e.g. for the electrooxidation of uric acid. The higher electrocatalytic properties of newly prepared surfaces were recognized, including the surfaces prepared with the repeated growth treatments in the 1:1 mixed solution of CTAB:HMT.

In contrast, the surface prepared with a previous growth treatment, i.e., using the growth solution contained CTAB without HMT, exhibited the lower electrocatalytic properties in comparison with the cases with CTAB/HMT. Even after loading higher amount of Au treated with the growth solution of CTAB followed by that of CTAB/HMT, the electrocatalytic properties were similar to that prepared with the growth solution of CTAB only. Hence, the difference in the final treatments, i.e., CTAB vs. CTAB/HMT, might affect the electrocatalytic properties. At least, the amount of the Au loading was found to be not a governing factor of the electrocatalysis.

The reasons of the better electrocatalytic properties of the AuNP-modified ITO electrodes prepared with the growth treatment with CTAB/HMT are not clear at moment. Because smaller AuNPs is known to have better catalytic performances with the supports of oxides, some merits of the smaller size might be manifested in the electrochemical oxidation also. However, due to the resolutions of the FE-SEM measurements, more focused observation of the local areas was difficult to observe the states of the outer-surfaces and boundaries of AuNPs. At present, however, we could propose the present AuNP-attached electrodes as those having practical potentials to the electrocatalytic detections in electroanalysis. 


\section{Acknowledgements}

This work was supported in part by a Grant-in-Aid for Scientific Research from the Ministry of Education, Culture, Science, Sports and Technology, Japan, No. 20550074. 


\section{References}

[1] C. M. Welch, R. G. Compton, Anal. Bioanal. Chem. 384 (2006) 601.

[2] F. W. Campbell, R. G. Compton, Anal. Bioanal. Chem. 396 (2010) 241.

[3] M. Oyama, Anal. Sci. 26 (2010) 1.

[4] M. S. El-Deab, T. Okajima, T. Ohsaka, J. Electrochem. Soc. 150 (2003) A851.

[5] M. S. El-Deab, T. Sotomura, T. Ohsaka, J. Electrochem. Soc. 152 (2005) C1.

[6] X. Dai, R. G. Compton, Anal. Sci. 22 (2006) 567.

[7] Y. Ma, J. Di, X. Yan, M. Zhao, Z. Lu, Y. Tu, Biosens. Bioelectron. 24 (2009) 1480.

[8] K. R. Brown, M. J. Natan, Langumuir 17 (2001) 1713.

[9] Y. Jin, X. Kang, Y. Song, B. Zhang, G. Cheng, S. Dong, Anal. Chem. 73 (2001) 2843.

[10] W. Cheng, S. Dong, E. Wang, Anal. Chem. 74 (2002) 3599.

[11] W. Cheng, S. Dong, E. Wang, Langmuir 18 (2002) 9947.

[12] M. Kambayashi, J. Zhang, M. Oyama, Cryst. Growth Des. 5 (2005) 81.

[13] J. Zhang, M. Kambayashi, M. Oyama, Electrochem. Commun. 6 (2004) 683.

[14] J. Zhang, M. Kambayashi, M. Oyama, Electroanalysis 17 (2005) 408.

[15] J. Zhang, M. Oyama, Electrochem. Acta 50 (2004) 85.

[16] J. Zhang, M. Oyama, J. Electroanal. Chem. 577 (2005) 273.

[17] A. Ali Umar, M. Oyama, Cryst. Growth Des. 5 (2005) 599.

[18] A. Ali Umar, M. Oyama, Appl. Surf. Sci. 253 (2006) 2196.

[19] A. Ali Umar, M. Oyama, Appl. Surf. Sci. 253 (2006) 2933.

[20] G. Chang, J. Zhang, M. Oyama, K. Hirao, J. Phys. Chem. B, 2005, 109, 1204.

[21] G. Chang, M. Oyama, K. Hirao, J. Phys. Chem. B 110 (2006) 20362. 
[22] A. Ali Umar, M. Oyama, Cryst. Growth Des. 6 (2006) 818.

[23] L. Vayssieres, Adv. Mater. 15 (2003) 464.

[24] A. Ali Umar, M. Oyama, Cryst. Growth Des. 8 (2008) 1808.

[25] A. Ali Umar, M. Oyama, Cryst. Growth Des. 9 (2009) 1146.

[26] R. N. Goyal, M. Oyama, A. Sangal, S. P. Singh, Indian J. Chem. A 44 (2005)

945.

[27] P. Kannan, S. A. John, Anal. Biochem. 386 (2009) $65 .$.

[28] D. K. Smith, B. A. Korgel, Langmuir 24 (2008) 644.

[29] D. K. Smith, N. R. Miller, B. A. Korgel, Langmuir 25 (2009) 9518. 


\section{Figure Captions}

Figure 1. Typical FE-SEM images of AuNP-attached ITO surfaces prepare using the seed-mediated growth method. For the growth solution, the ratio of [CTAB]:[HMT] was changed to (A) 3:1, (B) 2:1, (C) 3:2, (D) 1:1, (E) 1:3 and (F) 3:17, keeping the sum amount ([CTAB]+[HMT] $)$ as $0.10 \mathrm{M}$.

Figure 2. Cyclic voltammograms of $1.0 \mathrm{mM}$ uric acid recorded with (a) a bare ITO electrode, (b) an AuNP-attached ITO electrode prepared with CTAB only, and (c) an AuNP-attached ITO electrode prepared with the 1:1 mixed solution of CTAB and HMT, whose surface image is Fig. 1D. Scan rate: $50 \mathrm{mVs}^{-1}$.

Figure 3. Typical FE-SEM images of AuNP-attached ITO surfaces prepare with the seed-mediated repeated-growth treatment. (A) The treatment with the 1:1 mixed growth solution of CTAB:HMT was repeated twice. (B) At first, the growth treatment was performed with the 1:1 mixed solution of CTAB:HMT, and then, the second was with the growth solution contained CTAB only.

Figure 4. Cyclic voltammograms of $1.0 \mathrm{mM}$ uric acid recorded with (a) an AuNP-attached ITO electrode prepared with the repeated growth treatment with the 1:1 mixed solution of CTAB:HMT twice, whose surface image is Fig. 3A, and (b) that prepared with the 1:1 mixed solution of CTAB:HMT first, and then with the growth solution contained CTAB only, whose surface image is Fig. 3B. The grey volammograms are the data in Fig. 2 for comparison. Scan rate: $50 \mathrm{mVs}^{-1}$. 
Figure 5. Typical FE-SEM images of AuNP-attached ITO surfaces prepare with the seed-mediated repeated-growth treatment. The treatment with the 1:1 mixed growth solution of CTAB:HMT was repeated (A) three times and (B) four times.

Figure 6. Cyclic voltammograms of $1.0 \mathrm{mM}$ uric acid recorded with an AuNP-attached ITO electrode prepared with the repeated growth treatment with the 1:1 mixed solution of CTAB:HMT for (a) three times and (b) four times. The grey volammograms are the data recorded with that prepared twice treatment and a bare ITO electrode. Scan rate: $50 \mathrm{mVs}^{-1}$. 

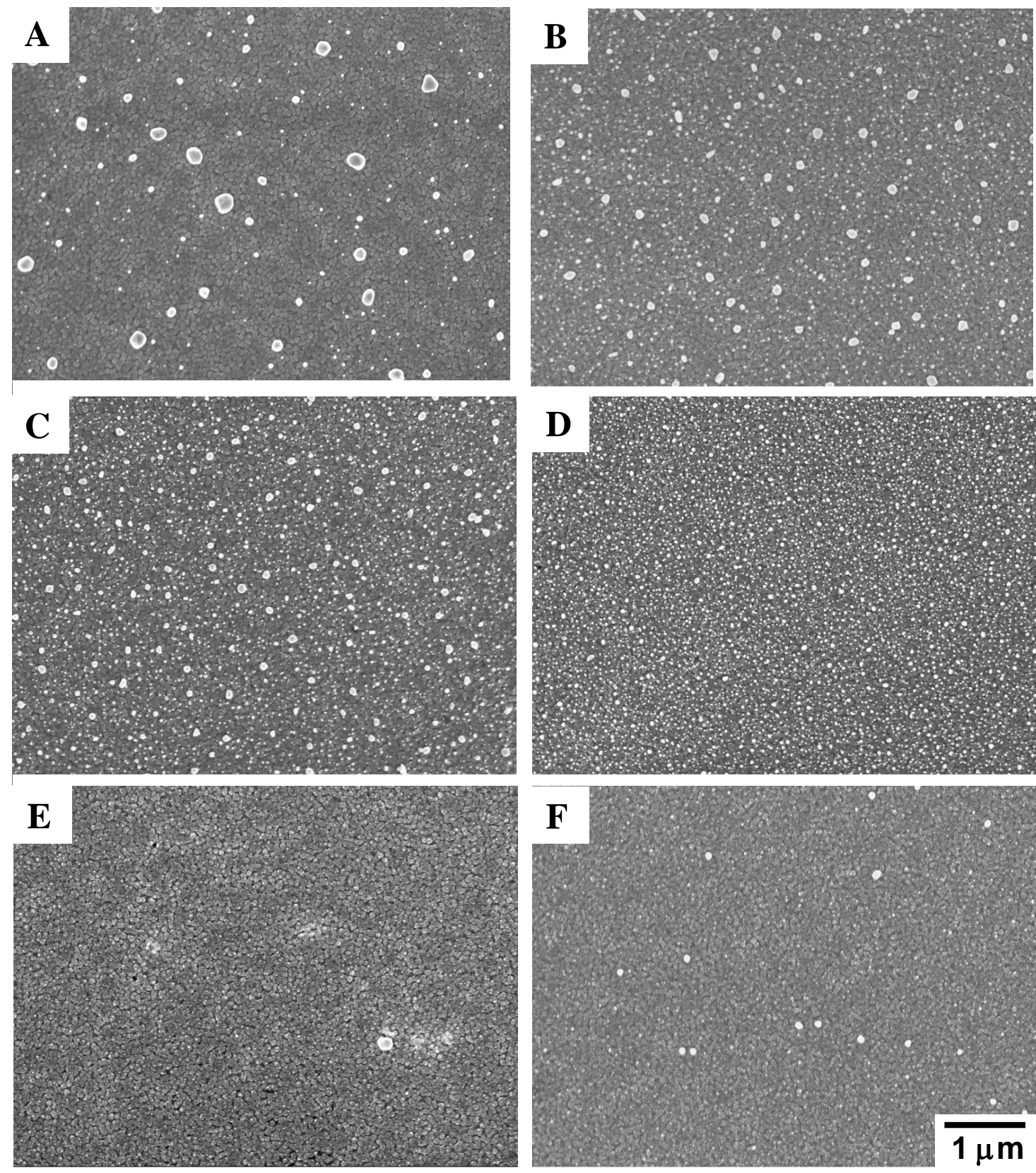

Figure 1. Typical FE-SEM images of AuNP-attached ITO surfaces prepare using the seed-mediated growth method. For the growth solution, the ratio of [CTAB]:[HMT] was changed to (A) 3:1, (B) 2:1, (C) 3:2, (D) 1:1, (E) 1:3 and (F) 3:17, keeping the sum amount ([CTAB]+[HMT]) as $0.10 \mathrm{M}$. 


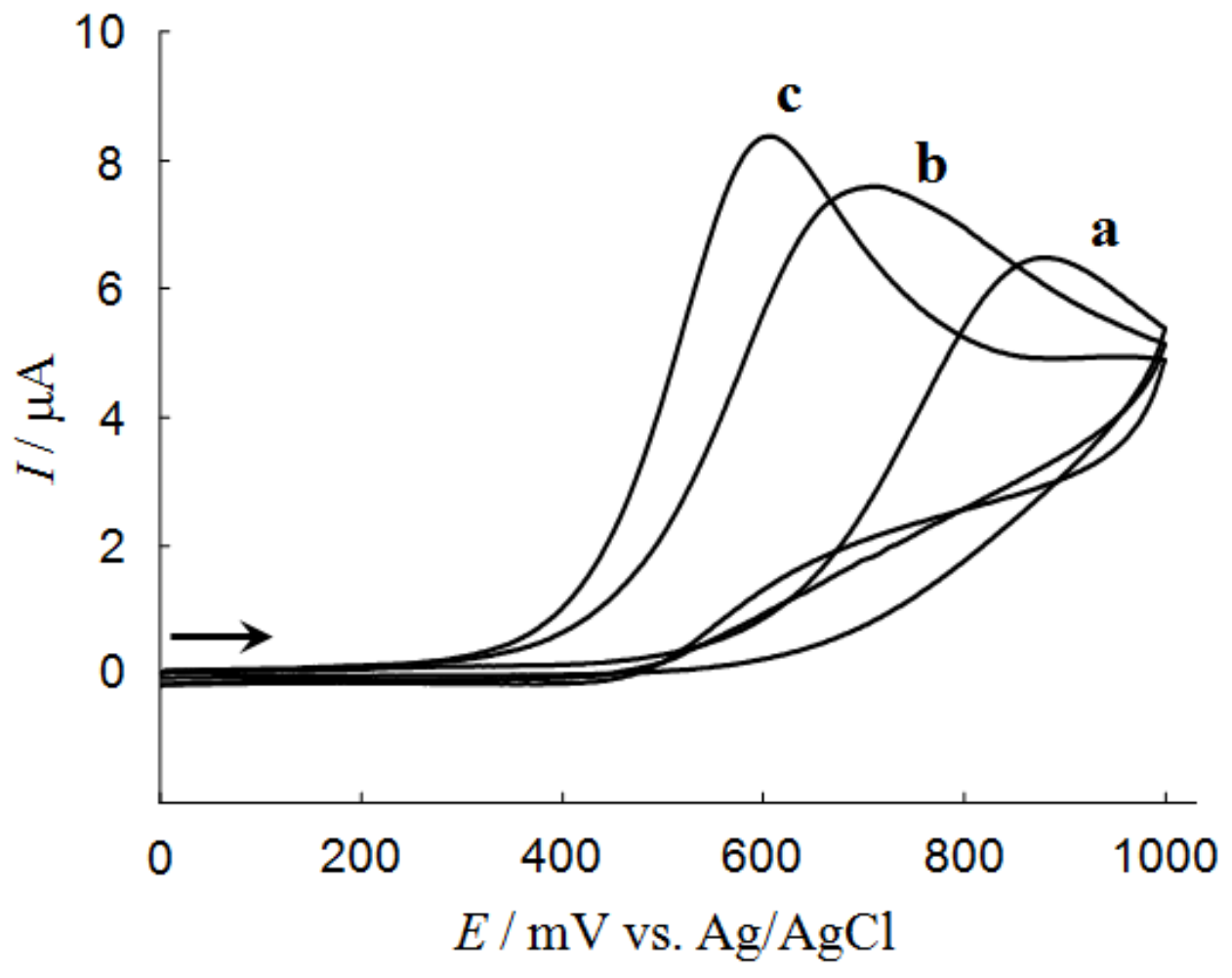

Figure 2. Cyclic voltammograms of $1.0 \mathrm{mM}$ uric acid recorded with (a) a bare ITO electrode, (b) an AuNP-attached ITO electrode prepared with CTAB only, and (c) an AuNP-attached ITO electrode prepared with the 1:1 mixed solution of CTAB and HMT, whose surface image is Fig. 1D. Scan rate: $50 \mathrm{mVs}^{-1}$. 


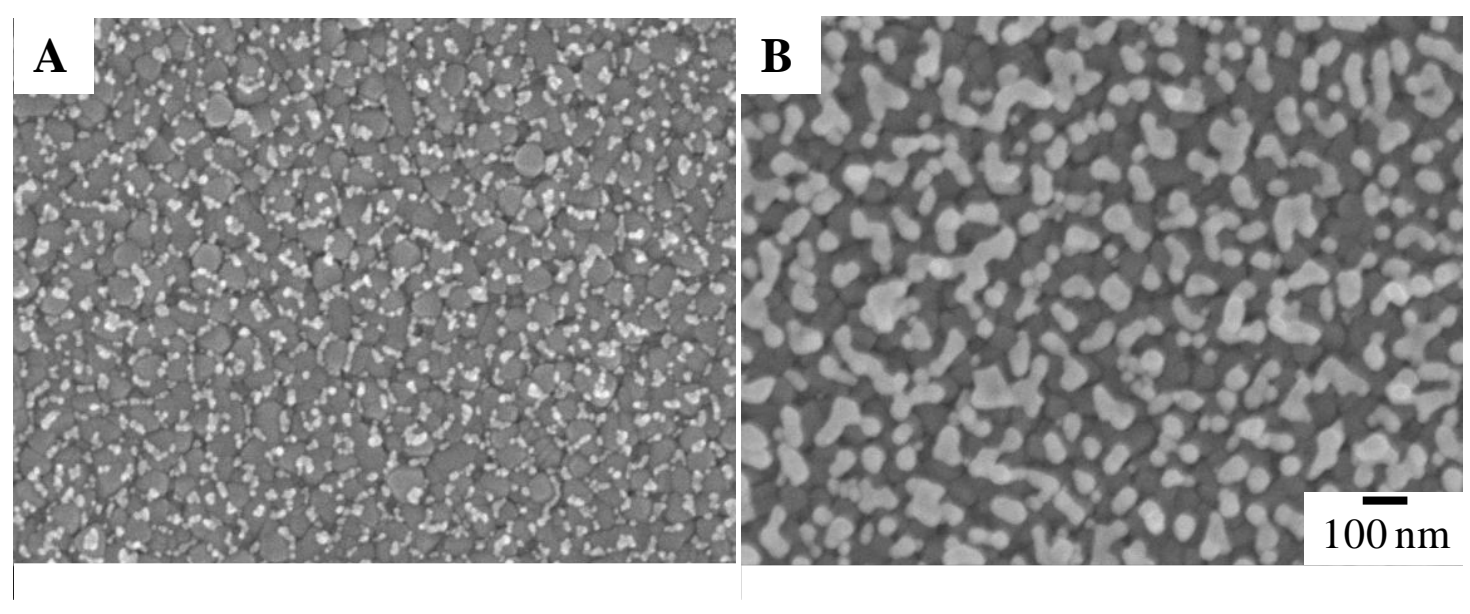

Figure 3. Typical FE-SEM images of AuNP-attached ITO surfaces prepare with the seed-mediated repeated-growth treatment. (A) The treatment with the 1:1 mixed growth solution of CTAB:HMT was repeated twice. (B) At first, the growth treatment was performed with the 1:1 mixed solution of CTAB:HMT, and then, the second was with the growth solution contained CTAB only. 


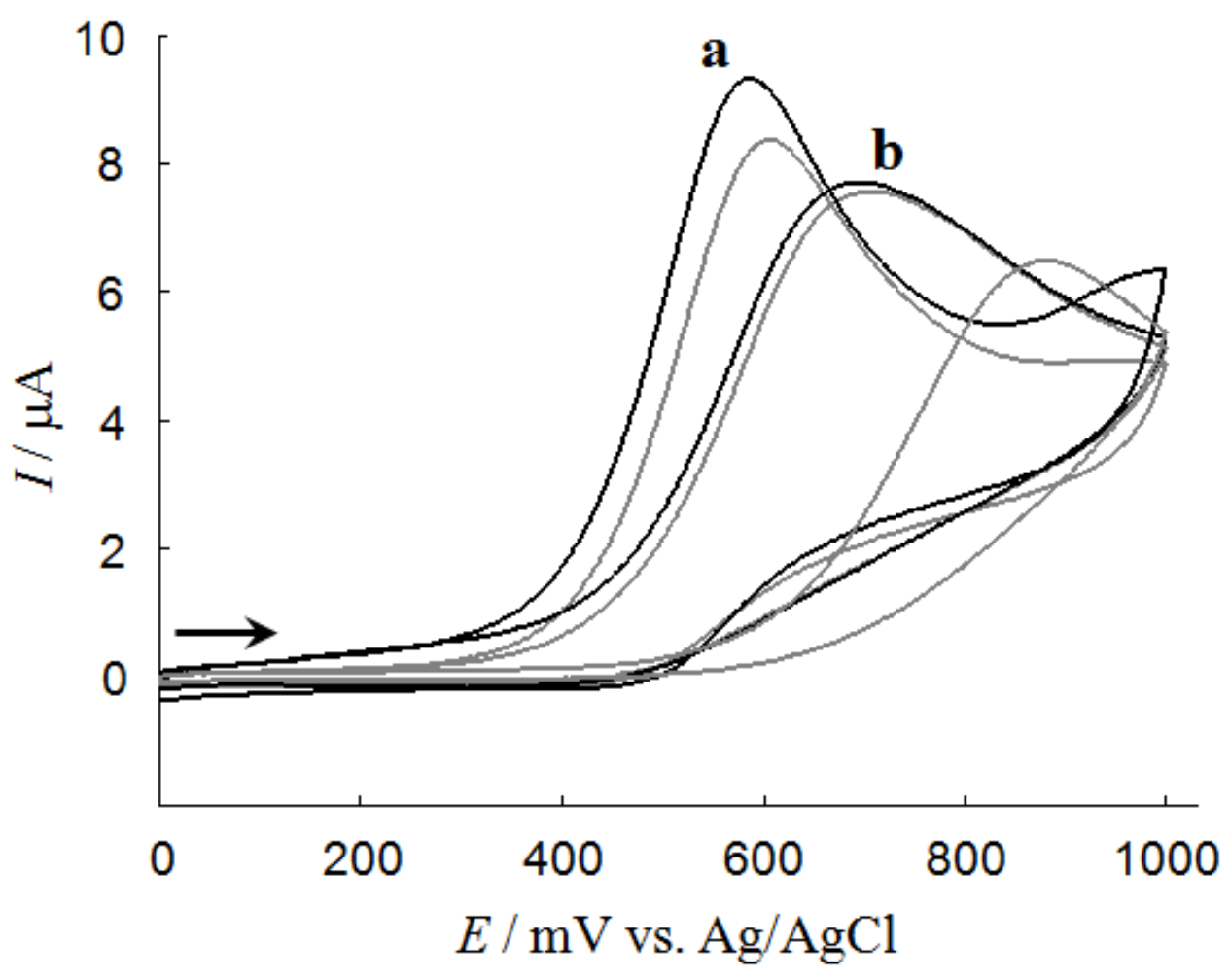

Figure 4. Cyclic voltammograms of $1.0 \mathrm{mM}$ uric acid recorded with (a) an AuNP-attached ITO electrode prepared with the repeated growth treatment with the 1:1 mixed solution of CTAB:HMT twice, whose surface image is Fig. 3A, and (b) that prepared with the 1:1 mixed solution of CTAB:HMT first, and then with the growth solution contained CTAB only, whose surface image is Fig. 3B. The grey volammograms are the data in Fig. 2 for comparison. Scan rate: $50 \mathrm{mVs}^{-1}$. 

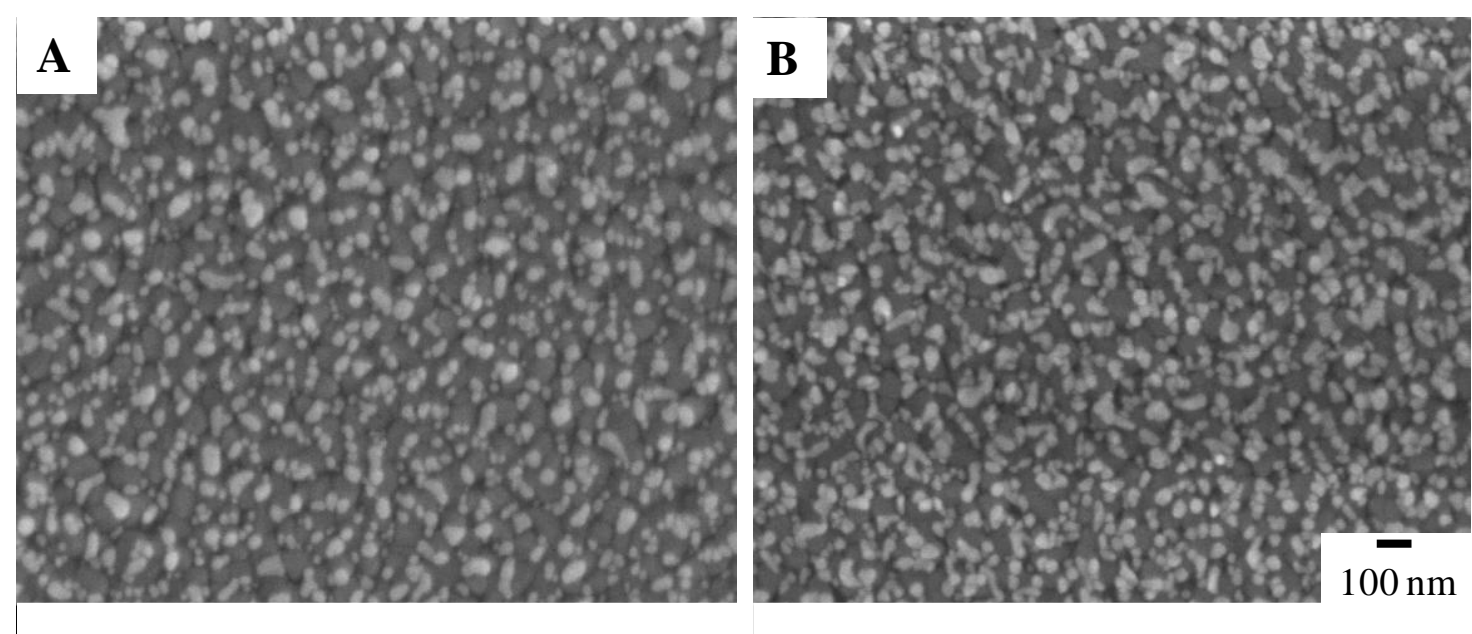

Figure 5. Typical FE-SEM images of AuNP-attached ITO surfaces prepare with the seed-mediated repeated-growth treatment. The treatment with the 1:1 mixed growth solution of CTAB:HMT was repeated (A) three times and (B) four times. 


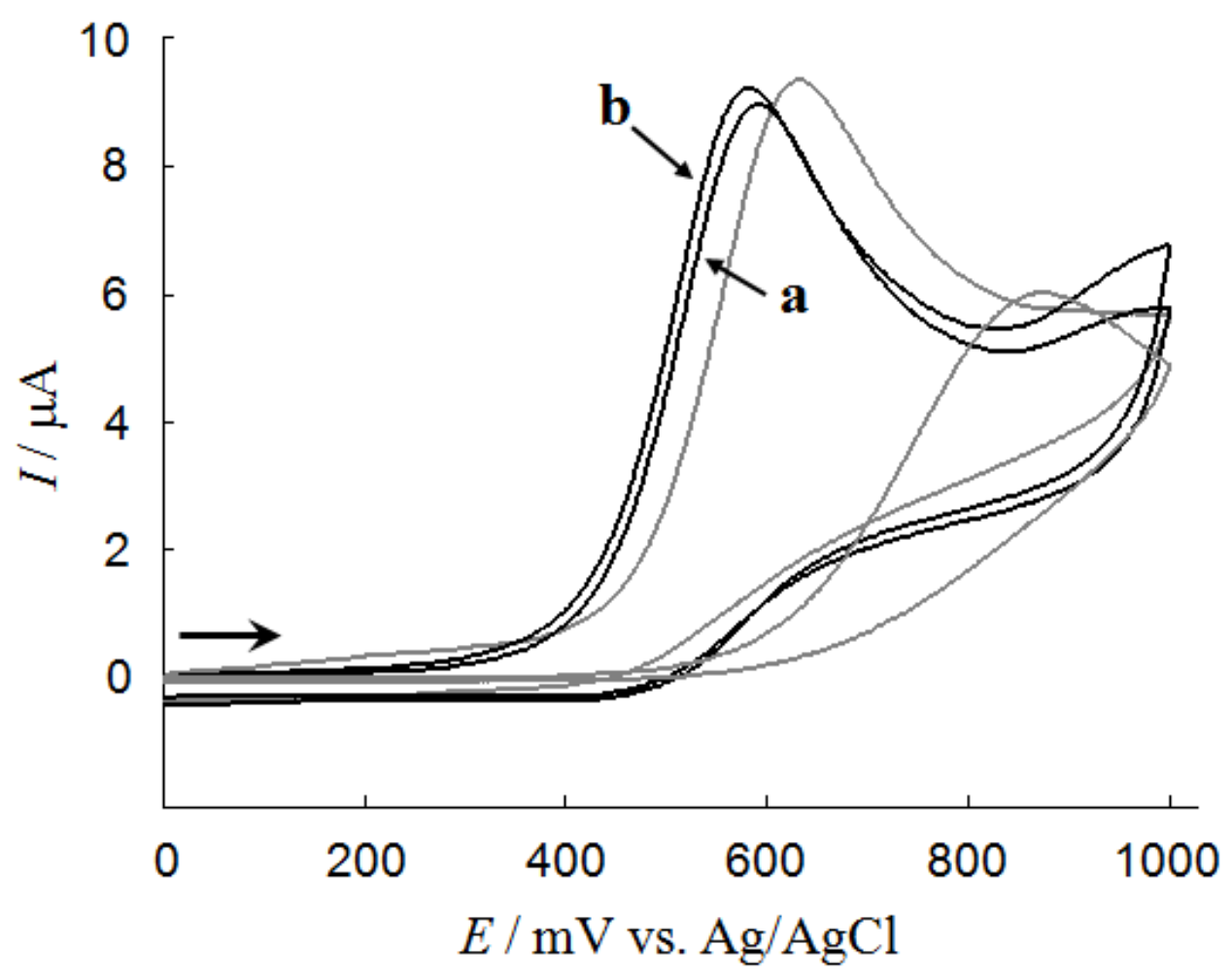

Figure 6. Cyclic voltammograms of $1.0 \mathrm{mM}$ uric acid recorded with an AuNP-attached ITO electrode prepared with the repeated growth treatment with the 1:1 mixed solution of CTAB:HMT for (a) three times and (b) four times. The grey volammograms are the data recorded with that prepared twice treatment and a bare ITO electrode. Scan rate: $50 \mathrm{mVs}^{-1}$. 